\title{
Molecular mapping of QTLs for plant type and earliness traits in pigeonpea (Cajanus cajan L. Millsp.)
}

Giriraj Kumawat ${ }^{1,3}$, Ranjeet S Raje ${ }^{2}$, Shefali Bhutani ${ }^{1}$, Jitendra K Pal ${ }^{1}$, Amitha SVCR Mithra ${ }^{1}$, Kishor Gaikwad ${ }^{1}$, Tilak R Sharma ${ }^{1}$ and Nagendra K Singh ${ }^{1 *}$

\begin{abstract}
Background: Pigeonpea is an important grain legume of the semi-arid tropics and sub-tropical regions where it plays a crucial role in the food and nutritional security of the people. The average productivity of pigeonpea has remained very low and stagnant for over five decades due to lack of genomic information and intensive breeding efforts. Previous SSR-based linkage maps of pigeonpea used inter-specific crosses due to low inter-varietal polymorphism. Here our aim was to construct a high density intra-specific linkage map using genic-SNP markers for mapping of major quantitative trait loci (QTLs) for key agronomic traits, including plant height, number of primary and secondary branches, number of pods, days to flowering and days to maturity in pigeonpea.

Results: A population of $186 \mathrm{~F}_{2: 3}$ lines derived from an intra-specific cross between inbred lines 'Pusa Dwarf' and 'HDM04-1' was used to construct a dense molecular linkage map of 296 genic SNP and SSR markers covering a total adjusted map length of $1520.22 \mathrm{cM}$ for the 11 chromosomes of the pigeonpea genome. This is the first dense intra-specific linkage map of pigeonpea with the highest genome length coverage. Phenotypic data from the $F_{2: 3}$ families were used to identify thirteen QTLs for the six agronomic traits. The proportion of phenotypic variance explained by the individual QTLs ranged from 3.18\% to 51.4\%. Ten of these QTLs were clustered in just two genomic regions, indicating pleiotropic effects or close genetic linkage. In addition to the main effects, significant epistatic interaction effects were detected between the QTLs for number of pods per plant.
\end{abstract}

Conclusions: A large amount of information on transcript sequences, SSR markers and draft genome sequence is now available for pigeonpea. However, there is need to develop high density linkage maps and identify genes/ QTLs for important agronomic traits for practical breeding applications. This is the first report on identification of QTLs for plant type and maturity traits in pigeonpea. The QTLs identified in this study provide a strong foundation for further validation and fine mapping for utilization in the pigeonpea improvement.

Keywords: Earliness, Pigeonpea, Plant height, QTL mapping, SSR marker, SNP marker

\section{Background}

Pigeonpea (Cajanus cajan L. Millsp.) is an economically important grain legume of the tropical and subtropical regions of the world. It is grown extensively in India, South-East Asia, East Africa, Latin America and the Caribbean, where its plays important role in the food and nutritional security of the people. It is a major

\footnotetext{
* Correspondence: nksingh@nrcpb.org

${ }^{1}$ National Research Centre on Plant Biotechnology, Indian Agricultural Research Institute, New Delhi 110012, India

Full list of author information is available at the end of the article
}

source of food protein to about $20 \%$ of the world population and is an abundant source of minerals and vitamins $[1,2]$. Further, it has significant usage as animal feed, fodder, firewood, thatching material and for improving soil structure and fertility. Globally, pigeonpea is cultivated in an area of 4.75 Mha with annual production of 3.68 MT and productivity of $706 \mathrm{~kg} / \mathrm{ha}$ [3]. The average productivity of pigeonpea has remained very low and stagnant over the last five decades. A major constraint to pigeonpea productivity is the low genetic potential of pigeonpea varieties that have low harvest index, poor plant type, long crop duration
C Biomed Central

(C) 2012 Kumawat et al.; licensee BioMed Central Ltd. This is an Open Access article distributed under the terms of the Creative Commons Attribution License (http://creativecommons.org/licenses/by/2.0), which permits unrestricted use, distribution, and reproduction in any medium, provided the original work is properly cited. 
and susceptibility to a host of biotic and abiotic stresses, besides socio-economic factors leading to poor crop management [4]. Exploitation of hybrid vigor, restructuring of plant type and early maturity are potential targets for increasing pigeonpea productivity per unit area and time $[5,6]$. Ideotype breeding is crucial for the suitability of a crop plant for modern farming practices, including traits for high harvest index and mechanical harvesting. It attempts to combine favorable QTLs for various component traits in a plant genotype [7]. Component traits of plant ideotype including plant height, number of branches, number of pods per plant and synchronous maturity play important role in shaping the plant architecture for high harvest index and mechanical harvesting. Early maturity is also needed for increasing cropping efficiency of the farming system. Although pigeonpea improvement through conventional breeding is going on, efforts for remodelling of pigeonpea plant type using genetic variability in the landraces and wild relatives with the help of modern biotechnological tools has not yet started.

The estimated size of the pigeonpea genome packed in its eleven chromosomes is about $853 \mathrm{Mb}$, which remained untouched by the genomics revolution for a long time $[8,9]$. Recently, a large number of simple sequence repeat (SSR) and single nucleotide polymorphism (SNP) markers have been developed under the Indo-US Agricultural Knowledge Initiative, creating opportunities for a large scale mapping of genes and quantitative trait loci (QTLs) for important agronomic traits [10-14]. Analysis of transcriptome and whole genome sequence using second generation high throughput sequencing technologies have made pigeonpea improvement amenable to molecular breeding [13,15-17]. Recently, an inter-specific linkage map of pigeonpea has been developed using 239 genomic SSR markers [14]. However, to our knowledge there is no published report on a dense intra-specific linkage map or QTL mapping of important agronomic traits in pigeonpea, except for limited studies using RAPD markers for tagging genes for Fusarium wilt resistance and plant type $[18,19]$. Earlier, SSR markers were used for the genetic diversity analysis of inbred lines and purity assessment of hybrids, but the level of polymorphism detected was very low [20]. The objective of present study was to develop an intra-specific high density framework linkage map of pigeonpea using genic SNP and SSR markers to identify QTLs for plant type and early maturity traits.

\section{Results}

\section{Intra-specific molecular linkage map}

Out of the total 595 SSR markers screened for parental polymorphism between Pusa Dwarf and HDM04-1, only 28 (4.7\%) were found polymorphic that amplified 29 SSR loci (ASSR42 produced two loci). Details of these 28 SSR markers showing polymorphism in our mapping population are provided in Additional file 1. With the GoldenGate SNP assays, 160 SNPs (10.41\%) were found polymorphic in the 1536-plex assay and 107 SNPs (13.93\%) were found polymorphic in the 768-plex assay (details in Additional file 2 and Additional file 3, respectively). Overall 296 marker loci $(10.17 \%)$ showed polymorphism and these were genotyped in all the $186 \mathrm{~F}_{2}$ plants. Total 280 marker loci (96.6\%) showed good agreement with the expected 1:2:1 segregation ratio when analysed using $X^{2}$ test $(P \geq 0.01)$. Significant segregation distortion was observed for the remaining 16 (3.4\%) marker loci. The direction of distortion for most of the loci was either towards the Pusa Dwarf allele or an excess of heterozygosity. A framework linkage map was constructed using all the 296 marker loci (Figure 1). Cosegregation analysis of markers resulted in the formation of eleven linkage groups at a cut-off recombination fraction of 0.35 , which is equal to the haploid chromosome number for pigeonpea (Cajanus cajan L.). No marker was found unlinked after linkage group assignment and ordering. The linkage map of 296 marker loci covered a total observed genome length of $1406.7 \mathrm{cM}$, with an adjusted genome length of $1520.22 \mathrm{cM}$ according to method 4 of Chakravarti et al. [21]. The average marker interval was $4.95 \mathrm{cM}$, providing a physical to genetic map ratio of $561.1 \mathrm{~kb} / \mathrm{cM}$ taking a genome size of $853 \mathrm{Mb}$ [8]. The eleven linkage groups included 16 to 55 marker loci. The linkage groups were numbered LG_Cc1 to LG_Cc11 based on the descending order of their total map length in cM. The highest number of 55 loci was present on linkage group LG_Cc1 covering an adjusted map length of $203.8 \mathrm{cM}$, followed by LG_Cc2 consisting of 36 markers covering an adjusted map length of $174.1 \mathrm{cM}$ and so on (Figure 1, Additional file 4). The shortest linkage group LG_Cc11 possessed 21 markers with an adjusted map length of $73.81 \mathrm{cM}$. The lowest number of 16 loci was mapped on the linkage group LG_Cc10 with an adjusted map length of $109.1 \mathrm{cM}$. There were only three gaps of larger than $30 \mathrm{cM}$ in the genome, one each on LG_Cc4, LG_Cc5 and LG_Cc7, with map distance of 35.5, 32.5 and $33 \mathrm{cM}$, respectively. Majority of the loci with distorted segregation (9/16 loci) were clustered on the linkage group LG_Cc4. The remaining seven loci with distorted segregation were randomly distributed on five other linkage groups, suggesting that it may be due to a chance factor. The recommended marker density for genome wide QTL scanning is an average marker interval of less than $10 \mathrm{cM}$ [22]. Thus the pigeonpea genetic map constructed in the present study with an average marker interval of $4.95 \mathrm{cM}$ was quite suitable for the mapping of QTLs for plant type and earliness traits.

\section{Phenotypic variation for the plant type and earliness traits}

Phenotypic evaluation of the plant type and earliness traits showed significant variability in the Pusa Dwarf/ 


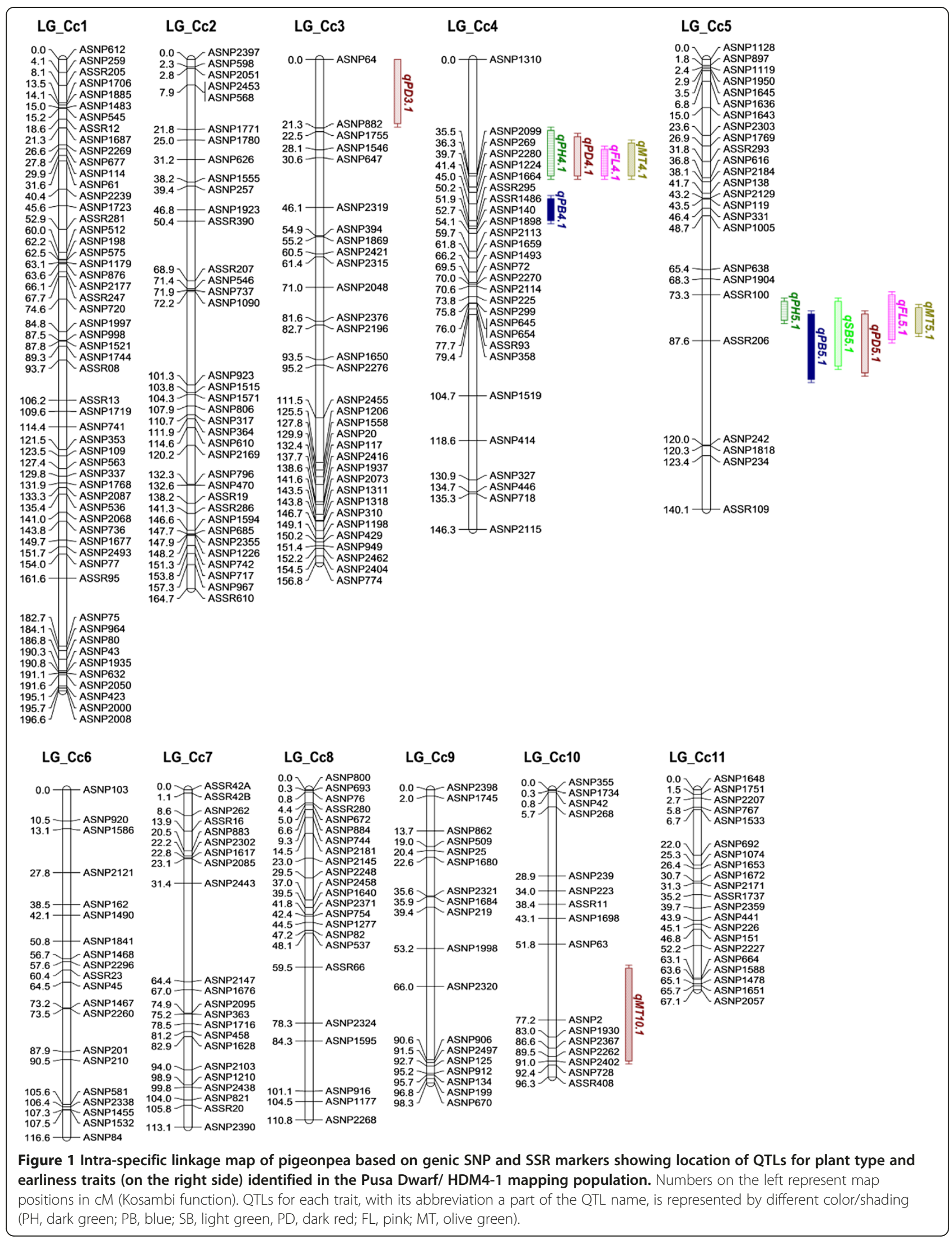


HDM04-1 mapping population (Table 1, Figure 2b). All the traits varied widely and their skewness values were less than 1.0, except for MT and SB. Frequency distribution for plant height and days to flowering showed bimodal distribution patterns, indicating involvement of major genes, whereas PB, PD and MT showed normal distribution patterns, suggesting involvement of multiple genes (Figure 2b). Transgressive segregation beyond both the parental means was observed for all the six traits, except for PB which did not show values higher than Pusa Dwarf. Pearson's correlation coefficients $(r)$ were calculated to see the relationships among various plant type and earliness traits. Significant positive correlations $(P<0.01)$ were observed for all the pair wise combinations of traits, except for $\mathrm{PH}$ which was significantly correlated with PB and FL only (Table 2). Highly significant positive correlations were observed for all the pair wise combinations of $\mathrm{PB}, \mathrm{SB}, \mathrm{PD}, \mathrm{FL}$ and $\mathrm{MT}$, which may be due to close linkage of genes for these traits or pleiotropic effect of common gene(s) controlling these traits.

\section{QTL analysis}

Thirteen significant QTLs were identified for the six agronomic traits analysed in this study using a full QTL model of the QTL Network software (Figure 1, Table 3). The proportion of phenotypic variation explained (PVE) by individual QTLs ranged from $3.18 \%$ to $51.4 \%$. Ten of these QTLs showed major effects with PVE of more than $10 \%$ each. However, there could be overestimation of PVE due to relatively smaller size of the mapping population. There was clustering of QTLs in the same or adjacent marker intervals on the linkage groups LG_Cc4 and LG_Cc5. This was expected from the highly significant positive correlations among the traits and is likely due to pleiotropy or very tight linkage between the genes (Table 2). Significant epistatic interactions were observed only with the QTLs for number of pods per plant.

\begin{tabular}{|c|c|c|c|c|c|}
\hline \multirow[t]{2}{*}{ Trait } & \multicolumn{2}{|c|}{ Parents } & \multicolumn{3}{|c|}{$F_{2: 3}$ Families } \\
\hline & Pusa Dwarf & HDM04-1 & Range & Mean \pm SD & Skewness \\
\hline $\mathrm{PH}$ & 87 & 119 & $59-160$ & $112.12 \pm 22.67$ & -0.552 \\
\hline PB & 19 & 5 & $4-16.6$ & $9.88 \pm 1.95$ & 0.199 \\
\hline SB & 13 & 0 & $0-25$ & $4.25 \pm 3.24$ & 2.33 \\
\hline PD & 120 & 24 & $6.6-170.9$ & $58.4 \pm 23.9$ & 0.853 \\
\hline $\mathrm{FL}$ & 106 & 66 & $59-116$ & $90.95 \pm 11.17$ & -0.623 \\
\hline MT & 158 & 116 & $110-208$ & $140.09 \pm 15.31$ & 1.223 \\
\hline
\end{tabular}

$\mathrm{PH}=$ plant height in $\mathrm{cm} ; \mathrm{PB}=$ number of primary branches/plant; $\mathrm{SB}=$ number of secondary branches/plant; $\mathrm{PD}=$ number of pods/plant; $\mathrm{FL}=$ days to flowering; $\mathrm{MT}=$ days to maturity.

\section{QTLs for plant type traits}

Two major additive effect QTLs, qPH4.1 and qPH5.1, were identified for plant height (Figure 1, Table 3). The $q$ PH4.1 was located on the linkage group LG_Cc4 in the marker interval ASNP1310-ASNP2099 with a peak $F$-value of 54.2 and PVE of $28.0 \%$, whereas, $q$ PH5.1 was located on linkage group LG_Cc5 in the marker interval ASSR100-ASSR206 with a peak F-value of 86.8 and PVE of $27.5 \%$. The qPH4.1 showed positive additive effect indicating that the allele for increasing plant height at this locus was contributed by Pusa Dwarf, whereas qPH5.1 showed a negative additive effect indicating that the allele for increasing plant height at this locus was contributed by HDM04-1. Thus, HDM04-1 has a dwarfing allele at the qPH4.1 locus whereas Pusa Dwarf has a dwarfing allele at the $q P H 5.1$ locus.

Two major QTLs were identified for the number of primary branches per plant, namely qPB4.1 located on the LG_Cc4 in the marker interval ASNP1664-ASSR295 and $q P B 5.1$ located on LG_Cc5 in the marker interval ASSR206-ASNP242 (Figure 1, Table 3). The qPB4.1 showed a peak $F$-value of 18.5 and PVE of $19.5 \%$, whereas $q P B 5.1$ showed a peak $F$-value of 10.9 and a PVE of $11.1 \%$. Both $q P B 4.1$ and $q P B 5.1$ showed positive additive effects indicating that the alleles for high number of primary branches were contributed by Pusa Dwarf at both the loci. A major additive effect QTL for number of secondary branches per plant, qSB5.1 was mapped on LG_Cc5 in the marker interval ASSR100-ASSR206 with peak $F$-value of 11 and PVE of $10.4 \%$. The positive allele at this locus for high number of SB was also contributed by Pusa Dwarf.

Two major additive effect QTLs and one minor QTL were detected for the number of pods per plant (Figure 1, Table 3). The first QTL, qPD4.1 was located on LG_Cc4 in the marker interval ASNP2099-ASNP269 with a peak $F$-value of 24.2 and PVE of $16.5 \%$. The second QTL, qPD5.1 was located on LG_Cc5 in the marker interval ASSR100- ASSR206 with a peak $F$-value of 31.3 and PVE of $18.9 \%$. Positive alleles for high pod number per plant at both these loci were contributed by the genotype Pusa Dwarf. In addition, a minor additive effect QTL qPD3.1 was mapped on LG_Cc3 in the marker interval ASNP64-ASNP882 with peak $F$-value of 9.53 and PVE of $3.18 \%$. Positive allele at this locus was also contributed by the variety Pusa Dwarf.

\section{QTLs for earliness traits}

Early maturity of crop plants is crucial for increasing the cropping efficiency of a farming system. One major and a minor QTL were detected for days to flowering, namely qFL4.1 located on LG_Cc4 in the marker interval ASNP1310-ASNP2099 and qFL5.1 located on LG_Cc5 in the marker interval ASSR100-ASSR206 (Figure 1, 


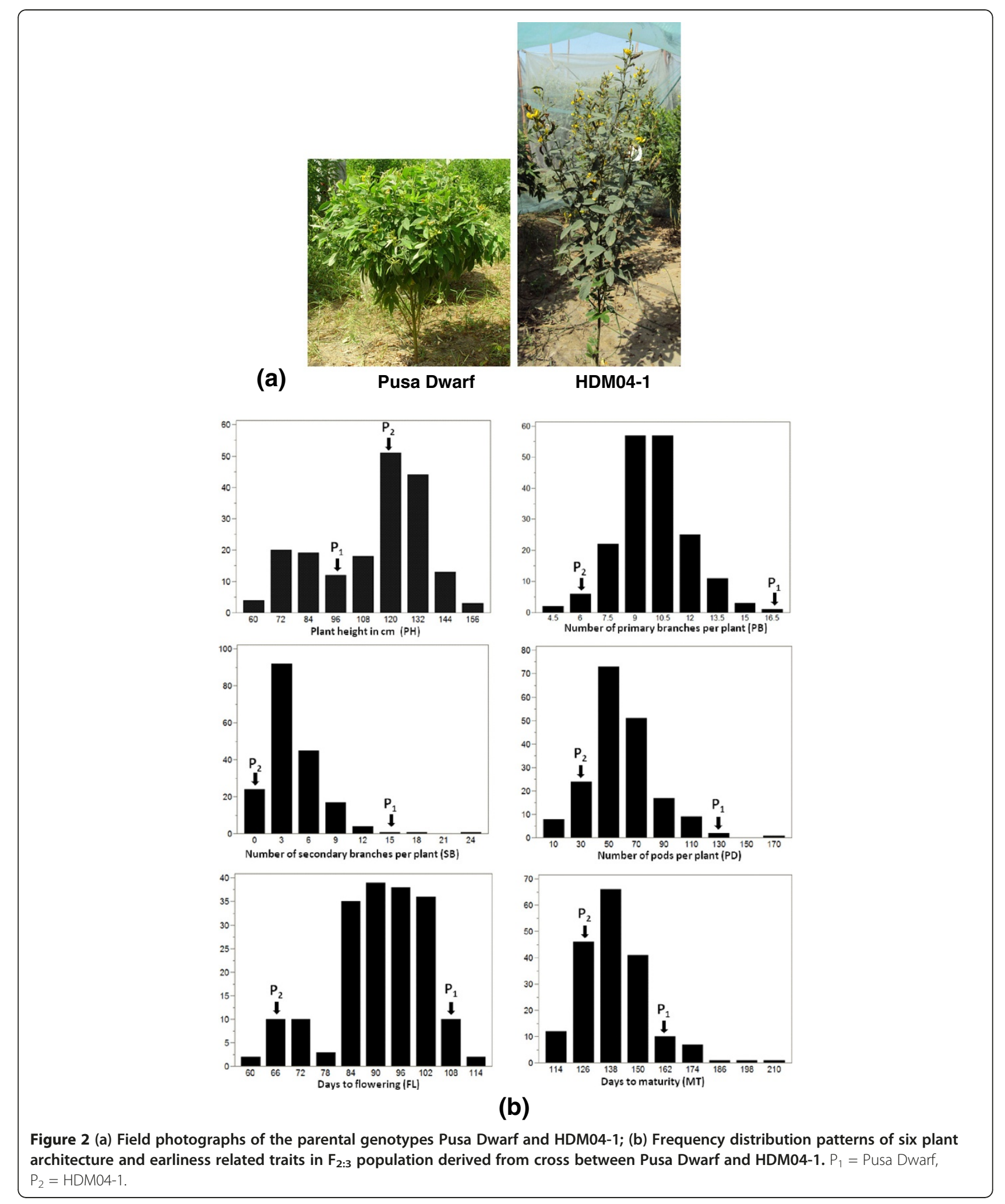

Table 3). The qFL4.1 was a highly significant QTL with peak $F$-value of 84.0 and PVE of $51.4 \%$, whereas $q F L 5.1$ was a relatively minor QTL with PVE of $8.7 \%$. Both the QTLs showed positive additive effect, therefore alleles for late flowering at both the loci were contributed by Pusa Dwarf. Two major additive effect QTLs and one minor QTL were identified for days to maturity. The two major QTLs, qMT4.1 and qMT5.1, were mapped on 
Table 2 Pearson's correlation coefficients among the six plant type and earliness traits analyzed in the Pusa Dwarf /HDM04-1 $F_{2: 3}$ families

\begin{tabular}{llllll}
\hline Traits* & PH & PB & SB & PD & FL \\
\hline PB & $0.270^{* *}$ & & & & \\
SB & -0.123 & $0.549^{* *}$ & & & \\
PD & -0.002 & $0.574^{* *}$ & $0.535^{* *}$ & & \\
FL & $0.193^{* *}$ & $0.517^{* *}$ & $0.366^{* *}$ & $0.631^{* *}$ & \\
MT & -0.112 & $0.409^{* *}$ & $0.359^{* *}$ & $0.545^{* *}$ & $0.816^{* *}$ \\
\hline
\end{tabular}

*Trait abbreviations are shown in Table 1; ${ }^{*}$ Significant at $P<0.01$.

LG_Cc4 and LG_Cc5, respectively. The qMT4.1 was located in the marker interval ASNP2099-ASNP269 with a peak F-value of 38.0 and PVE of $22.6 \%$. The qMT5.1 was located in the marker interval ASSR100-ASSR206 with a peak $F$-value of 49.9 and PVE of $25.9 \%$. In addition, a minor QTL for days to maturity, qMT10.1 was mapped on LG_Cc10 in the marker interval ASNP2262- ASNP2402, with peak $F$-value of 8.94 and PVE of $3.19 \%$. While the first two QTLs showed positive additive effects, indicating alleles for late maturity in Pusa Dwarf, the reverse was true for locus qMT10.1 with allele for late maturity coming from HDM04-1.

\section{Epistatic interaction and pleiotropic effects of QTLs}

Apart from the main effects, an epistatic interaction between different QTLs controlling the same trait was analyzed using a full-QTL model employing Markov Chain Monte Carlo algorithm of the QTL Network software. No significant epistatic effect was observed for $\mathrm{PH}$, $\mathrm{PB}, \mathrm{SB}, \mathrm{FL}$ and MT. However, for number of pods per plant, QTL qPD3.1 showed significant additive by additive, additive by dominance and dominance by dominance interactions with $q P D 5.1$ (Figure 3). The epistatic interactions between $q P D 3.1$ and $q P D 5.1$ contributed to $6.79 \%$ of the total phenotypic variance as indicated by the heritability estimate of the epistatic allele by QTL Network- 2.1 (Table 4). The thirteen QTLs identified in this study for the plant type and earliness traits were located in four genomic regions of pigeonpea chromosomes LG_Cc3, LG_Cc4, LG_Cc5 and LG_Cc10 (Figure 1). Ten of these QTLs were clustered in just two locations on the linkage groups LG_Cc4 and LG_Cc5. Since phenotypic values for most of the traits were significantly correlated with each other, the co-localization of QTLs was not entirely unexpected. To test whether this was due to pleiotropic effect of a single locus or close genetic linkage between different genes, the two QTL regions were analyzed for significance of pleiotropy using the Qgene software. The multiple trait multiple interval mapping (MMIM) function identified significant pleiotropic effects at both the QTL clusters as indicated by significantly high LOD of pleiotropy and LOD of the joint effect (Table 5).

\section{Discussion}

Semi-dwarf plant type and synchronous early maturity are two very important agronomic traits for enhancing crop productivity per unit area and time by harnessing high harvest index and cropping efficiency. Grain legumes, particularly pigeonpea has lagged behind in the development of high yielding cultivars due to lack of basic information on the genomic regions and genes associated with these traits. In the present study, a dense molecular linkage map of pigeonpea was constructed using genic-SNP and genic-SSR markers to identify the QTLs or genomic regions associated with the plant type and early maturity traits. The genic-SNP markers

Table 3 QTLs for plant type and earliness traits identified by QTL Network 2.1 using Pusa Dwarf / HDM04-1 F $2: 3$ population

\begin{tabular}{|c|c|c|c|c|c|c|c|}
\hline Trait & QTL Name & Marker interval & Position (cM) & $F$ value & PVE(\%) & Additive effect & Dominance effect \\
\hline \multirow[t]{2}{*}{$\mathrm{PH}$} & qPH4.1 & ASNP1310-ASNP2099 & 30.0 & 54.2 & 28.0 & 21.8 & 7.92 \\
\hline & qPH5.1 & ASSR100-ASSR206 & 79.3 & 86.8 & 27.5 & -20.14 & 14.94 \\
\hline \multirow[t]{2}{*}{ PB } & qPB4.1 & ASNP1664-ASSR295 & 49.1 & 18.5 & 19.5 & 1.26 & -0.03 \\
\hline & qPB5.1 & ASSR206-ASNP242 & 87.6 & 10.9 & 11.1 & 0.81 & 0.19 \\
\hline SB & qSB5.1 & ASSR100-ASSR206 & 85.3 & 11.0 & 10.4 & 1.64 & -0.49 \\
\hline \multirow[t]{3}{*}{ PD } & qPD3.1 & ASNP64-ASNP882 & 10.0 & 9.53 & 3.18 & 7.81 & -13.20 \\
\hline & qPD4.1 & ASNP2099-ASNP269 & 35.5 & 24.2 & 16.5 & 17.75 & 11.24 \\
\hline & qPD5.1 & ASSR100-ASSR206 & 85.3 & 31.3 & 18.9 & 15.86 & -7.35 \\
\hline \multirow[t]{2}{*}{$\mathrm{FL}$} & qFL4.1 & ASNP1310-ASNP2099 & 34.0 & 84.0 & 51.4 & 14.4 & 5.3 \\
\hline & qFL5.1 & ASSR100-ASSR206 & 79.3 & 26.3 & 8.7 & 5.54 & -2.8 \\
\hline \multirow[t]{3}{*}{ MT } & qMT4.1 & ASNP2099-ASNP269 & 35.5 & 38.0 & 22.6 & 11.72 & 3.10 \\
\hline & qMT5.1 & ASSR100-ASSR206 & 81.3 & 49.9 & 25.9 & 13.09 & -6.28 \\
\hline & qMT10.1 & ASNP2262- ASNP2402 & 89.5 & 8.94 & 3.19 & -4.13 & -3.61 \\
\hline
\end{tabular}




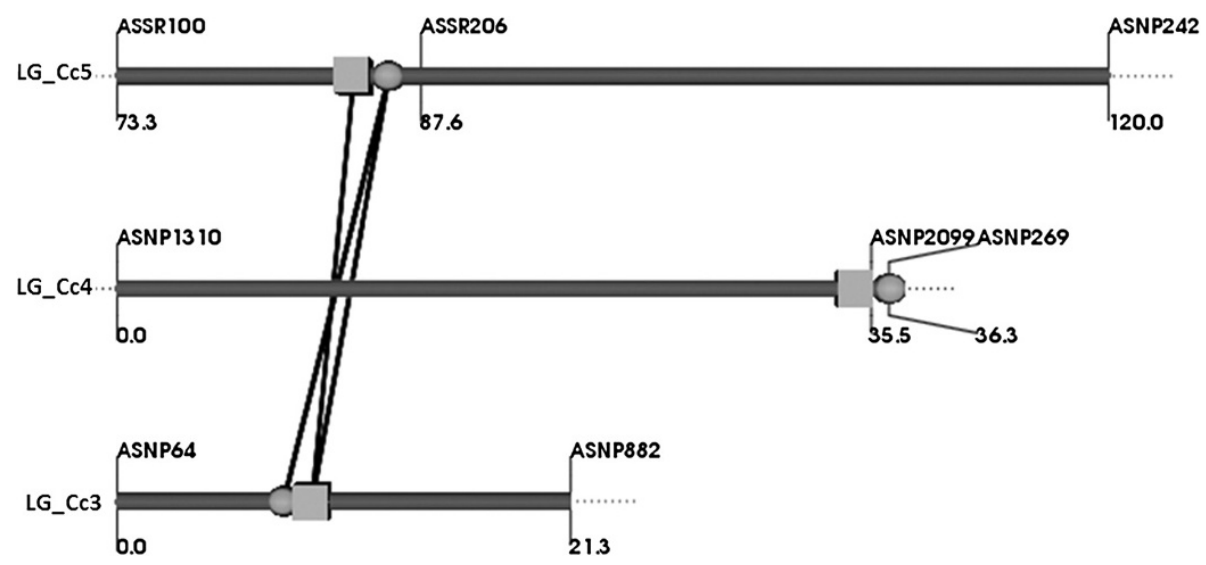

Figure 3 Graphic representation of QTLs showing interaction effects for the number of pods per plant in Pusa Dwarf /HDM04-1 $\mathrm{F}_{2: 3}$ population. Circles represent QTLs with only additive effect while squares represents QTLs with only dominant effect. Connecting lines indicate significant interactions between the main effect QTLS.

showed significantly higher level of polymorphism $(11.58 \%)$ than the genic-SSR markers (4.7\%). Although this may be partly due to higher resolving power of the SNP assays, it will be prudent to use SNP markers for high density genetic mapping in crops like pigeonpea with narrow genetic base. This is due to high abundance of the SNP markers and high success rate of the GoldenGate SNP genotyping assays. In contrast, small allelic size differences of the SSR markers are not easily resolved by common agarose gel electrophoresis, although capillary electrophoresis can resolve these small size differences and produce higher polymorphism success rates [14]. The genic markers used in this study were developed from a comprehensive transcriptome assembly dataset; therefore this linkage map represents a random gene set of the pigeonpea genome [13]. Earlier a BES-SSR based integrated inter-specific genetic map of C. cajan $\times C$. scarabaeoides has been reported that has 239 loci with a genome map length of $930.9 \mathrm{cM}$ [14]. The inter-specific mapping population comprised of $79 \mathrm{~F}_{2}$ plants and a large proportion of the markers (63.5\%) showed distorted segregation as compared to the present intra-specific population of $186 \mathrm{~F}_{2}$ plants with only $3.4 \%$ markers showing distorted segregation. The present map is the first high density intra-specific molecular linkage map of pigeonpea based on genic-SNP and genic-SSR markers and covers a much higher genome map length of $1520.22 \mathrm{cM}$. This is due to a larger population size and better pairing and crossing over between the chromosomes of two varieties of the same species. Furthermore, the present map represents expressed regions of the pigeonpea genome; therefore it will be highly useful for comparative genomics and synteny studies. However, genic markers represent mostly the euchromatic regions of the genome; hence heterochromatin and other repeat regions may be underrepresented, leading to large physical gaps between genic markers spanning these regions. There were three gaps of larger than $30 \mathrm{cM}$ in the present genetic map, one each on LG_Cc4, LG_Cc5 and LG_Cc7, Presence of large gaps may lead to failure in detection of QTLs in the gap regions. Interestingly, large gaps were also observed in the recently developed genomic-SSR based consensus genetic map on LG8, LG10 and LG11 [14]. Although a direct comparison of the two maps was not possible due to lack of common markers, a non-uniform distribution of markers was apparent in both the maps.

This is the first report in pigeonpea on the mapping of QTLs for plant architecture and maturity time related traits which are very important for the development of superior varieties. Thirteen QTLs were identified, including ten QTLs having major effects with PVE of higher than $10 \%$. The number of QTLs identified in a bi-parental

Table 4 Epistatic interactions between QTLs for number of pods per plant in the Pusa Dwarf/HDM04-1 $F_{2: 3}$ population of pigeonpea

\begin{tabular}{|c|c|c|c|c|c|c|c|c|}
\hline Locus 1 & Flanking markers $^{a}$ & LG (position) $^{b}$ & Locus 2 & Flanking markers $^{a}$ & LG (position) $^{\mathbf{b}}$ & Gene effect $^{c}$ (value) & $P$-value & PVE \\
\hline \multirow[t]{3}{*}{ qPD3.1 } & ASNP64-ASNP882 & $3(10.0)$ & qPD5.1 & ASSR100-ASSR206 & $5(85.3)$ & AA (7.8) & 0.015 & 1.58 \\
\hline & & & & & & $\mathrm{AD}(-5.1)$ & 0.251 & 3.74 \\
\hline & & & & & & DD (17.54) & 0.017 & 1.47 \\
\hline
\end{tabular}

a Markers flanking the of $F$-value peak interval for the QTL.

${ }^{b}$ Location (in $\mathrm{CM}$ ) of the highest point of $F$-value peak for the QTL.

${ }^{c} A A$, additive by additive; $A D$, additive by dominance; $D D$, dominance by dominance interaction. 
Table 5 Pleiotropic effects of co-localized QTLs on two linkage groups identified by QGene 4.0 software

\begin{tabular}{lllll}
\hline Linkage group & Marker interval of QTL cluster (Map position) & Traits of co-located QTL & LOD of pleiotropy & LOD of joint effect \\
\hline LG_CC4 & ASNP1310-ASNP2099 (0-35 cM) & PH, PD, FL, MT & 12 & 35 \\
LG_CC5 & ASSR206-ASNP242 (87.6-120 cM) & PH, PB, SB, PD, FL, MT & 39 & 480 \\
\hline
\end{tabular}

mapping population depends on the number of trait controlling loci having contrasting alleles between the two parents [23]. The high PVE exhibited by the QTLs for many of the traits indicates involvement of segregating alleles of only a few critical genes leading to a large change in the plant architecture and maturity time of the two parents, particularly for $\mathrm{PH}$ and FL (Figure 2, Table 1). However, a possibility of the overestimation of QTL effects due to small population size, sometimes referred to as the Beavis effect, could not be ruled out in this preliminary investigation [24-26]. For further investigation of possible Beavis effects and validation of QTLs under different environments and genetic backgrounds, RIL mapping populations are being developed.

Plant height is an important agronomic and yield contributing trait for which two major QTLs were identified. Pusa Dwarf allele at the locus qPH4.1 increased plant height by $21.8 \mathrm{~cm}$, whereas HDM04-1 allele at the locus qPH5.1 increased plant height by $20.1 \mathrm{~cm}$. The two dwarfing genes will be very useful for the modification of plant type in pigeonpea. A high number of primary and secondary branches and number of pods per plant are also important yield contributing traits for which QTLs were collocated in the same marker interval. The QTLs $q$ PB4.1 and $q$ PB5.1 both showed positive additive effects with Pusa Dwarf alleles contributing to higher number of primary branches. The qSB5.1 also showed positive additive effect with Pusa Dwarf allele contributing to a high number of secondary branches. The number of pods per plant directly contributes to higher grain yield and two major additive effect QTLs, qPD4.1 and qPD5.1 were identified, both showing positive additive effect with Pusa Dwarf alleles enhancing 17.75 pods and 15.86 pods per plant, respectively. A minor QTL on linkage group LG_Cc3, qPD3.1 also showed positive additive effect with Pusa Dwarf allele increasing 7.81 pods per plant. Thus, Pusa Dwarf has several positive yield contributing traits that can be utilized in the pigeonpea improvement using markerassisted breeding. It will be useful to combine the tallness allele of Pusa Dwarf at the qPH4.1 locus with the dwarfing allele from Pusa Dwarf at the qPH5.1 locus to obtain a semi-dwarf plant type with high number of primary branches, determinate growth habit and large number of pods per plant.

Pigeonpea has a large variation in the flowering and maturity time; therefore genetic mapping of these traits has direct implications for the development of short duration high yielding pigeonpea varieties. Two additive effect QTLs were identified for days to flowering (qFL4.1 and qFL5.1) and alleles from the early flowering genotype HDM04-1 at these loci decreased the time of flowering by 14.4 days and 5.54 days, respectively. Three QTLs were identified for days to maturity, the two major loci qMT4.1 and qMT5.1 showed positive additive effects with alleles from Pusa Dwarf increasing the maturity time by 11.72 and 13.09 days, respectively. In addition, HDM04-1 allele at a minor locus qMT10.1 increased the maturity time by 4.13 days. Hence, combining HDM041 alleles at the qMT5.1 locus with Pusa Dwarf allele at the qMT10.1 locus will reduce the days to maturity by about 17 days. Due to pleiotropic effects of the two QTL loci on height and maturity traits, it will be necessary to have the Pusa Dwarf allele at the qMT.4.1 locus which is also associated with large number of primary branches and number of pods per plant. However, these are our preliminary observations, further validation of the QTLs at multiple environments and in different genetic background are needed for molecular breeding applications.

Epistatic interaction between $q P D 3.1$ and $q P D 5.1$ loci for number of pods was significant, accounting for $6.79 \%$ of the phenotypic variance. It illustrates the need for considering both additive and epistatic effects for devising effective molecular breeding strategy. Ten of the thirteen QTLs identified in this study were co-localized in just two genomic regions on linkage groups LG_Cc4 and LG_Cc5. The clustering of QTLs can be explained either by the presence of different tightly linked genes or by pleiotropic effects of a single regulatory gene [27]. Clustering of QTLs for different agronomic traits has been reported in many crops including soybean, common bean and rice [28-31]. Statistical analysis using Qgene software [32] devised for this purpose revealed that co-location of QTLs was due to significant pleiotropic effects at each of the two loci. The pleiotropy is possibly due to involvement of genes for the synthesis of phytohormones regulating common signaling pathways of the traits during plant development. Plant height was positively correlated with the number of primary branches, number of pods, flowering time and maturity time. As the flowering and maturity times increase, the plant gets more time for increased plant height and vegetative growth due to indeterminate growth habit.

Unfortunately, large genetic intervals were observed for some QTL regions, leading to poor resolution of closely linked QTLs and preventing accurate estimation of PVE. Although many of the QTLs identified in this 
study were mapped to small genomic regions of 5-15 cM (qPH5.1, qPB4.1, qFL5.1, qMT5.1) and were closely flanked by other polymorphic markers, their utilization in MAS is possible after validation in different genetic backgrounds and environments. Therefore, QTL validation and fine mapping is the next step towards successful application of these findings in MAS.

\section{Conclusions}

The work presented here describes the first intraspecific dense molecular linkage map of pigeonpea largely based on genic-SNP markers. It is also the first report on QTL mapping of agronomically important traits in pigeonpea, including plant height, number of branches and pods per plant and maturity time. These results provide strong basis for further investigation on validation and fine mapping of the identified QTLs for plant type and earliness traits, which would help in developing high-yielding early-maturing varieties of pigeonpea for food and nutritional security in the semiarid tropical and sub-tropical countries.

\section{Methods}

\section{Plant material}

A population of $186 \mathrm{~F}_{2: 3}$ lines derived from a cross between inbred lines 'Pusa Dwarf' and 'HDM04-1' was used. Pusa Dwarf has a compact dwarf plant type, determinate growth habit, late maturity and higher number of primary and secondary branches and number of pods per plant as compared to HDM04-1 which has a medium plant height, indeterminate growth habit, early maturity with lower numbers of primary branches and pods per plant (Figure 2a, Table 1). Original seeds of the two parental lines were obtained from the Indian Agricultural Research Institute, New Delhi.

\section{Experimental details and trait phenotyping}

The $186 \mathrm{~F}_{2: 3}$ families and the two parental lines were planted in single rows with $60 \times 20 \mathrm{~cm}$ spacing in two replications in a randomized block design (RBD) at the research farm of Indian Agricultural Research Institute, New Delhi in June 2009. The field management followed standard agricultural practices. Observations were recorded on plant height $(\mathrm{PH}$ in $\mathrm{cm})$, number of primary branches per plant $(\mathrm{PB})$, number of secondary branches per plant (SB), number of pods per plant (PD), days to flowering (FL) and days to maturity (MT). Ten plants from the middle of each row were used for trait scoring. Days to flowering was scored as number of days from the date of sowing on which the first flower opened, whereas days to maturity was scored as number of days from the date of sowing when $80 \%$ of the pods turned yellow. All the plant type traits viz. PH, PB, SB and PD were recorded at maturity.

\section{Genotyping and construction of linkage maps}

Genomic DNA was isolated from 2 g leaf sample of each of the $\mathrm{F}_{2}$ plants using cetyl trimethyl ammonium bromide (CTAB) method with minor modifications [33]. Total 595 SSR markers consisting of 550 genic-SSR [13] and 45 genomic-SSR [4,34] were used for polymorphism survey between the parental lines Pusa Dwarf and HDM04-1. The SSR markers were PCR amplified and separated by electrophoresis in high resolution 4\% Metaphor agarose gels or $8 \%$ polyacrylamide gels. Multiplexed genic-SNP markers, including a 1536-plex and a 768-plex assays employing Illumina's GoldenGate technology, were used for SNP genotyping of the $F_{2}$ population. The SNPs were identified by aligning high quality transcriptome shotgun assembly (TSA) sequence contigs of pigeonpea varieties Asha (35,204 contigs, NCBI Ac. no. EZ647865-EZ683068) and UPAS 120 (30,147 contigs, NCBI Ac. no. EZ617718EZ674864) [13], using Lasergene SeqMan Pro $^{\text {Tm }}$ Version 8.0.12 software and designated ASNP1-ASNP2498. From this SNP list, two multiplex assays of 1536-plex and 768plex were designed using Illumina's GoldenGate assay design tool (www.illumina.com). Genomic DNAs (250 ng per sample) were used as templates for the SNP genotyping using Illumina's bead array technology according to the manufacturer's protocol [35]. Allelic segregation at each of the marker loci was analysed for deviation from the expected 1:2:1 ratio in the $\mathrm{F}_{2}$ population using $\chi^{2}$ test and linkage maps were constructed using Mapdisto version 1.7.5 software [36]. Seriation-II method was used for ordering of the loci with the criteria SARF for ordering and ripple. A cut-off recombination value of 0.35 and threshold LOD score of 3.0 was used. The Kosambi function was used for the estimation of map distances [37]. Adjusted genetic map lengths (in cM) were calculated using method 4 of Chakravarti et al. [20,38].

\section{Statistical analysis and QTL mapping}

Statistical analysis of the phenotypic data was performed using SAS software (SAS Institute Inc. USA). Pearson's correlation coefficients were calculated for pairwise trait combinations. The QTL analysis was performed using means of the phenotypic trait values from two replications with QTL Network version 2.1 software employing the full-QTL model [39]. The test window size was set to 5 $\mathrm{cM}$ with a walk speed of $2 \mathrm{cM}$ and a cut-off probability of 0.05 for deciding the significance of the QTL. F-value threshold for declaring a QTL significant was determined by permutation tests using 1,000 reiterations [40]. The QTL main effects and their epistasic interactions were analysed by the full-QTL model using a Markov Chain Monte Carlo algorithm. Graphical presentation of the linkage groups (LGs) and the QTLs were obtained using MapChart version 2.2 [41]. Pleiotropy of co-localized 
QTLs was tested by multiple trait multiple interval mapping (MMIM) function of the Qgene 4.3.8 software [32].

\section{Additional files}

Additional file 1: Table showing details of 28 polymorphic SSR markers used in the construction of linkage map of pigeonpea from the Pusa Dwarf/HDM04-1 population.

Additional file 2: Table showing SNP positions [in bracket] and flanking sequences (about 50 bases each side of the SNPs) in the 1536-plex Illumina Goldengate assay, with the SNP Id, TSA (transcript shotgun assembly) contig Id of the sequence from variety 'Asha' (NCBI GenBank Ac. no. EZ647865-EZ683068) and SNP quality scores based on Illumina SNP assay design tool.

Additional file 3: Table showing SNP positions [in bracket] and flanking sequences (about 50 bases each side of the SNPs) in the 768-plex Illumina Goldengate assay, with the SNP Id, TSA (transcript shotgun assembly) contig Id of the sequence from variety 'Asha' (NCBI GenBank Ac. no. EZ647865-EZ683068) and SNP quality scores based on Illumina SNP assay design tool.

Additional file 4: Table showing observed and adjusted map lengths of the 11 linkage groups of pigeonpea.

\section{Abbreviations}

BLAST: Basic local alignment search tool; cM: CentiMorgan; EST: Expressed sequence tag; EST-SSR: An SSR obtained from a CDNA sequence located in EST; ESTP: Expressed sequence tag polymorphism; GO: Gene Ontology; LG: Linkage group; LOD: Log10 of the likelihood odds ratio; NCBI: National Center for Biotechnology Information; PCR: Polymerase chain reaction; QTL: Quantitative trait locus; SNP: Single nucleotide polymorphism; SSR: Simple sequence repeat.

\section{Competing interests}

The authors declare that they have no competing financial interests.

\section{Authors' contributions}

GK carried out SSR genotyping, linkage map construction, phenotyping, QTL mapping and drafted the manuscript. RSR, GK, KG and TRS contributed to the design of the study and mapping population development. SB developed SNP GoldenGate assays. JKP and ASVCRM carried out SNP genotyping. NKS conceived and supervised the study as well as finalized the manuscript. All authors have read and approved the final version of the manuscript.

\section{Acknowledgements}

This study was financially supported by the NPTC project of the Indian Council of Agricultural Research (ICAR). GK acknowledges fellowship support from the Department of Biotechnology, Government of India.

\section{Author details}

${ }^{1}$ National Research Centre on Plant Biotechnology, Indian Agricultural Research Institute, New Delhi 110012, India. ${ }^{2}$ Division of Genetics, Indian Agricultural Research Institute, New Delhi 110012, India. ${ }^{3}$ Present address: Directorate of Soybean Research, Indore, Madhya Pradesh 452001, India.

Received: 8 May 2012 Accepted: 28 September 2012

Published: 8 October 2012

\section{References}

1. Saxena KB, Kumar RV, Rao PV: Pigeonpea nutrition and its improvement. In Quality Improvement in Field Crops. Edited by Basra AS, Randhawa IS. New York: Food Products Press; 2002:227-260.

2. Thu TT, Mai TTX, Dewaele E, Farsi S, Tadesse Y, Angenon G, Jacobs M: In vitro regeneration and transformation of pigeonpea (Cajanus cajan (L.) Millsp.). Mol Breed 2003, 11:159-168.

3. FAOSTAT. 2012. http://faostat.fao.org.
4. Odeny DA, Jayashree B, Ferguson M, Hoinsington D, Crouch J, Gebhardt C: Development, characterization and utilization of microsatellite markers in pigeonpea. Plant Breed 2007, 126:130-136.

5. Saxena KB, Sharma D: Pigeonpea genetics. In The pigeonpea. Edited by Nene YL, Hall SD, Sheila VK. Wallingford (UK): CAB International; 1990:137-158.

6. Saxena KB: Genetic improvement of pigeonpea-a review. Trop Plant Biol 2008, 1:159-178.

7. Wu RL: Genetic mapping of QTLs affecting tree growth and architecture in Populus: implication for ideotype breeding. Theor Appl Genet 1998, 96:447-457.

8. Greilhuber J, Obermayer R: Genome size variation in Cajanus cajan (Fabaceae): a reconsideration. Plant Syst Evol 1998, 212:135-141.

9. Yang S, Pang W, Harper J, Carling J, Wenzl P, Huttner E, Zong X, Kilian A: Low level of genetic diversity in cultivated pigeonpea compared to its wild relatives is revealed by diversity arrays technology (DArT). Theor Appl Genet 2006, 113:585-595.

10. Varshney RK, Close TJ, Singh NK, Hoisington DA, Cook DR: Orphan legume crops enter the genomics era. Curr Opin Plant Biol 2009, 12:202-210

11. Varshney RK, Penmetsa RV, Dutta S, Kulwal PL, Saxena RK, Datta S, Sharma TR, Rosen B, Carrasquilla-Garcia N, Farmer AD, Dubey A, Saxena KB, Gao J, Fakrudin B, Singh MN, Singh BP, Wanjari KB, Yuan M, Srivastava RK, Kilian A, Upadhyaya HD, Mallikarjuna N, Town CD, Bruening GE, He G, May GD, McCombie R, Jackson SA, Singh NK, Cook DR: Pigeonpea genomics initiative (PGI): an international effort to improve crop productivity of pigeonpea (Cajanus cajan L.). Mol Breed 2010, 26:393-408.

12. Raju NL, Gananesh BN, Lekha P, Jayashree B, Pande S, Hiremath PJ, Byregowda M, Singh NK, Varshney RK: The first set of EST resource for gene discovery and marker development in pigeonpea (Cajanus cajan L.). BMC Plant Biol 2010, 10:45.

13. Dutta S, Kumawat G, Singh BP, Gupta DK, Singh S, Dogra V, Gaikwad K, Sharma TR, Raje RS, Bandhopadhya TK, Datta S, Singh MN, Bashasab F, Kulwal P, Wanjari K, Varshney RK, Cook DR, Singh NK: Development of genic-SSR markers by deep transcriptome sequencing in pigeonpea [Cajanus cajan (L.) Millspaugh]. BMC Plant Biol 2011, 11:17.

14. Bohra A, Dubey A, Saxena RK, Penmetsa RV, Poornima KN, Kumar N, Farmer AD, Srivani G, Upadhyaya HD, Gothalwal R, Ramesh S, Singh D, Saxena K, Kishor PB, Singh NK, Town CD, May GD, Cook DR, Varshney RK: Analysis of BAC-end sequences (BESs) and development of BES-SSR markers for genetic mapping and hybrid purity assessment in pigeonpea, (Cajanus spp.). BMC Plant Biol 2011, 11:56.

15. Dubey A, Farmer A, Schlueter J, Cannon SB, Abernathy B, Tuteja R, Woodward J, Shah T, Mulasmanovic B, Kudapa H, Raju NL, Gothalwal R, Pande S, Xiao Y, Town CD, Singh NK, May GD, Jackson S, Varshney RK: Defining the transcriptome assembly and its use for genome dynamics and transcriptome profiling studies in pigeonpea (Cajanus cajan L.). DNA Res 2011, 18:153-164.

16. Singh NK, Gupta DK, Jayaswal PK, Mahato AK, Dutta S, Singh S, Bhutani S, Dogra V, Singh BP, Kumawat G, Pal JK, Pandit A, Singh A, Rawal H, Kumar A, Prashat GR, Khare A, Yadav R, Raje RS, Singh MN, Datta S, Fakrudin B, Wanjari KB, Kansal R, Dash PK, Jain PK, Bhattacharya R, Gaikwad K, Mohapatra T, Srinivasan R, Sharma TR: The first draft of the pigeonpea genome sequence. J. Plant Biochem. Biotechnol. 2011, 21:98-112.

17. Kudapa H, Bharti AK, Cannon SB, Farmer AD, Mulaosmanovic B, Kramer R, Bohra A, Weeks NT, Crow JA, Tuteja R, Shah T, Dutta S, Gupta DK, Singh A, Gaikwad K, Sharma TR, May GD, Singh NK, Varshney RK: A comprehensive transcriptome assembly of pigeonpea (Cajanus cajan L.) using sanger and second-generation sequencing platforms. Mol Plant 2012,1-9. doi:10.1093/mp/ssr111.

18. Kotresh H, Fakrudin B, Punnuri SM, Rajkumar BK, Thudi M, Paramesh H, Lohithaswa $\mathrm{H}$, Kuruvinashetti MS: Identification of two RAPD markers genetically linked to a recessive allele of a Fusarium wilt resistance gene in pigeonpea (Cajanus cajan L. Millsp.). Euphytica 2006, 149:113-120.

19. Dhanasekar P, Dhumal KN, Reddy KS: Identification of RAPD markers linked to plant type gene in pigeonpea. Indian J Biotechnol 2010, 9:58-63.

20. Chakravarti A, Lasher L, Reefer J: A maximum likelihood method for estimating genome length using genetic linkage data. Genetics 1991, 128:175-182. 
21. Saxena RK, Saxena K, Varshney RK: Application of SSR markers for molecular characterization of hybrid parents and purity assessment of ICPH 2438 hybrid of pigeonpea [Cajanus cajan (L.) Millspaugh]. Mol Breed 2010, 26:371-380.

22. Doerge RW: Mapping and analysis of quantitative trait loci in experimental populations. Nat Rev 2002, 3:43-52.

23. Mackay I, Powell W: Methods for linkage disequilibrium mapping in crops. Trends Plant Sci 2007, 12:57-63.

24. Beavis WD: QTL Analyses: Power, precision and accuracy. In Molecular dissection of complex traits. Edited by Paterson AH. Boca Raton: CRC Press; 1998:145-162.

25. Xu S: Theoretical basis of the Beavis effect. Genetics 2003, 165:2259-2268.

26. Raghavan C, Collard BCY: Effect of small mapping population sizes on reliability of quantitative trait locus (QTL) mapping. African J Biotechnol 2012, 11:10661-10674.

27. Aastveit $\mathrm{AH}$, Aastveit $\mathrm{K}$ : Effects of genotype-environment interactions on genetic correlations. Theor Appl Genet 1993, 86:1007-1013.

28. Mansur LM, Orf JH, Chase K, Jarvik T, Cregan PB, Lark KG: Genetic mapping of agronomic traits using recombinant inbred lines of soybean. Crop Sci 1996, 36:1327-1336.

29. Beattie AD, Larsen J, Michaels TE, Pauls KP: Mapping quantitative trait loci for a common bean (Phaseolus vulgaris L.) ideotype. Genome 2003, 46:411-422.

30. Blair MW, Iriarte G, Beebe S: QTL analysis of yield traits in an advanced backcross population derived from a cultivated Andean wild common bean (Phaseolus vulgaris L.) cross. Theor Appl Genet 2006, 112:1149-1163.

31. Wang P, Zhou G, Cui K, Li Z, Yu S: Clustered QTL for source leaf size and yield traits in rice (Oryza sativa L.). Mol Breed 2012, 29:99-113.

32. Joehanes R, Nelson JC: QGene 4.0, an extensible Java QTL-analysis platform. Bioinforma 2008, 24:2788-2789.

33. Murray MG, Thompson WF: Rapid isolation of high molecular weight plant DNA. Nucleic Acid Res 1980, 8:4321-4325.

34. Odeny DA, Jayashree B, Gebhardt C, Crouch J: New microsatellite markers for pigeonpea (Cajanus cajan (L.) millsp.). BMC Res Notes 2009, 2:35.

35. Shen R, Fan JB, Campbell D, Chang W, Chen J, Doucet D, Yeakley J, Bibikova M, Wickham Garcia E, McBride C, Steemers F, Garcia F, Kermani BG, Gunderson K, Oliphant A: High-throughput SNP genotyping on universal bead arrays. Mutat Res 2005, 573:70-82.

36. Lorieux M: MapDisto: fast and efficient computation of genetic linkage maps. Mol Breed 2012, 30:1231-1235.

37. Kosambi DD: The estimation of map distance from recombination values. Ann Eugen 1944, 12:172-175.

38. Echt CS, Saha S, Krutovsky KV, Wimalanathan K, Erpelding JE, Liang C, Nelson CD: An annotated genetic map of loblolly pine based on microsatellite and CDNA markers. BMC Genet 2011, 12:17.

39. Yang J, Hu C, Hu H, Yu R, Xia Z, Zhu J: QTLNetwork: mapping and visualizing genetic architecture of complex traits in experimental populations. Bioinforma 2008, 24:721-723.

40. Doerge RW, Churchill GA: Permutation tests for multiple loci affecting a quantitative character. Genetics 1996, 142:285-294.

41. Voorrips RE: Mapchart: software for the graphical presentation of linkage maps and OTLs. J Hered 2002, 93:77-78.

doi:10.1186/1471-2156-13-84

Cite this article as: Kumawat et al: Molecular mapping of QTLs for plant type and earliness traits in pigeonpea

(Cajanus cajan L. Millsp.). BMC Genetics 2012 13:84.

\section{Submit your next manuscript to BioMed Central and take full advantage of:}

- Convenient online submission

- Thorough peer review

- No space constraints or color figure charges

- Immediate publication on acceptance

- Inclusion in PubMed, CAS, Scopus and Google Scholar

- Research which is freely available for redistribution

Submit your manuscript at www.biomedcentral.com/submit
Ciomed Central 